\title{
Virtual reconstruction of historical landscapes: a 3D environment to contextualise data in space and time
}

\author{
Margherita Azzari a, *, Vincenzo Bologna a , Pauline Deguy ${ }^{\text {a }}$ \\ ${ }^{a}$ University of Florence, Department of History, Archaeology, Geography, Arts and Performing Arts, margherita.azzari@unifi.it , \\ vincenzo.bologna@unifi.it, pauline.deguy@unifi.it \\ * Corresponding author
}

Keywords: 3D GIS, 3D Geometry Visualizer, Historical Landscapes reconstruction

\begin{abstract}
:
The paper describes the results of a project with the aim of contributing to territorial knowledge taking into consideration the dynamics connected to time and historical values that currently persist as effects in the forms of landscape.
\end{abstract}

The reconstruction of historical landscapes is motivated by the need to single out informative layers belonging to different time phases, to be able to read each individual landscape, to identify the characterizing elements and the invariants to be preserved. These reconstructions also allow to contextualize cultural assets in space and time as well as to produce and spread knowledge through effective communication strategies, which can be effectively used in planning procedures, educational contexts and touristic applications in order to enhance the value and knowledge of territory through a deeper land evaluation screening and/or through the simulation of future scenarios.

Technologies like GIS and 3D rendering are very useful in this field of interest, as they allow dealing with sets of historical data highlighting the transformation processes and producing highly communicative immersive environments. Using GIS technology, all data can be geo-referenced, sources which differ in time of realization and in intrinsic characteristics can be integrated, and easier to use vector layers can be extrapolated, so that the analysis and comparison of all information provided by each source can be performed. The process of reconstruction is based on a wide selection of cartographical, iconographical and documentary sources enabling the philological reconstruction of landscape. In the case of a disappeared landscape, the 3D rendering can be used to bring it back and to give awareness and responsibility to the local population about the need to preserve their cultural heritage.

To fulfil this aim was designed a software to manage geographic dimensioned data, from various survey methods, to make them compatible with GIS systems. The aim is to design an efficient, flexible and scalable working tool for the generation of geometries with $\mathrm{Z}$ dimension to reconstruct historical landscapes in different time phases and contextualise cultural assets in space and time. The data processed by this platform are immediately usable in a 3D GIS, allowing to treat historical data sets, highlighting transformation processes and producing highly communicative immersive environments.

In the absence of a software suitable for the purpose, we have designed a platform capable of importing geolocalized data from various sources via spreadsheets (batch import) and via input. The adopted database is PostgreSQL with the PostGIS extension fully compatible with the most common GIS platforms (for example: QGIS and ESRI ArcGIS).

The platform has the following features:

- $\quad$ archiving and normalization of the data produced

- $\quad$ possibility of generating 3D geometries (with $\mathrm{Z}$ dimensions) to be associated with the data

- lemmatization of data related to interpretations and investigation methodologies

- tools for exporting data to the ArcGIS geodatabase via shapefile.

We started defining a workflow with all the specialized teams which produced all geolocalized data (e.g: structural analysis, GPR, archival research and drilling). This phase was crucial in order to better define all software specifications and the business logic itself. Regarding the choice of geometry and synthesis of the imported data, we have identified specific typologies to associate (PointZ for archival and documentary data sources; MultiPointZ: for the 
boreholes survey sources; PolygonZ: for the data coming from cartographical and iconographic sources. These 3D geometries are needed to deliver organized data in a GIS environment.

Scripts have been developed for the stable and automated import of the data coming from the pre-existing datasets in order to create the corresponding sheets on the project database. The development of the scripts ensures that over time the import of the cards is schedulizable and minimizes the filing activities between databases.

The import scripts involve loading sets based on an Excel (XLS, XLSX) or CSV structure with corresponding fields established by each team.

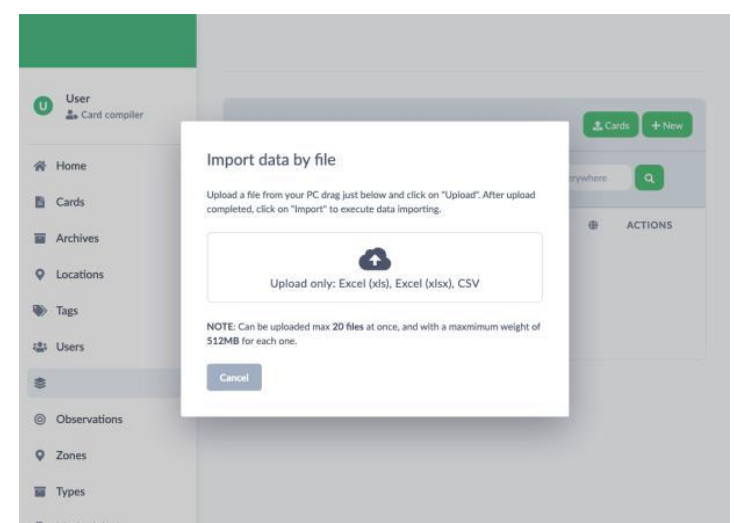

Figure 1. Example of WebApp importing interface

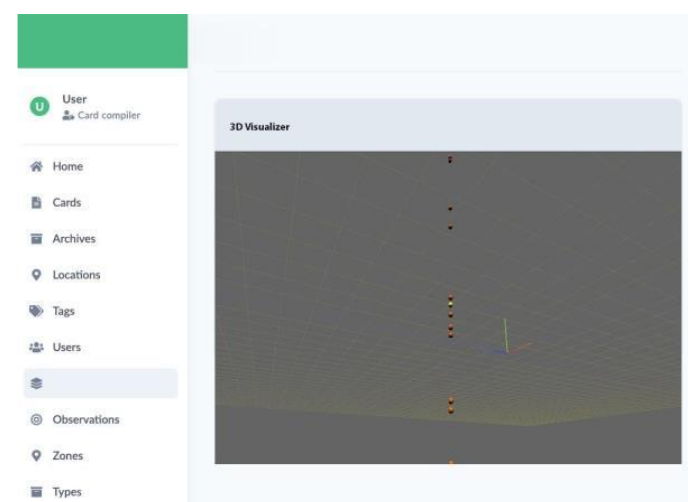

Figure 2. 3D Visualizer Preview

Regarding the interpretation of time phases of the data, even before being exported for use in a GIS environment, the $3 \mathrm{D}$ visualization tool of the points in the space is being designed and developed, which will allow to work on the interpretation in a completely visual and systematic way, ensuring the distribution in space and the geospatial relationship of all georeferenced data. This tool will allow to visualize all the layers distinguished by colour according to the typology of the data, their already assigned interpretation.

The last feature being designed concerns the export of all sorted data that has been processed in the software. We identified Shapefile export as the most flexible data interchange tool. The tool we are developing can create on-the-fly a shapefile with selected georeferenced and interpreted data directly within the WebApp interface, depending on the selection of the interpretation associated with the data. In other words, all geometries generated for a homogeneous dataset (same type of geometry) for a specific interpretation associated with that data are collected. Once the Shapefile is generated, it is downloaded directly from the browser as a zip file containing the three files provided (dbf, shp and shx). These files can be imported directly into ArcGIS and contain not only the expected geometries but also the table with all the specific source card information.

The designed software allows to manage data from different acquisition methods in an efficient 3D environment to virtually recreate historical disappeared landscapes. The export of the data processed by the software using shapefiles and specific interpretative categories makes it possible to create a realistic DTM by interpolation, since it is based on archaeological and archival data and all the subsurface surveys carried out. The DTMs can then be cross-referenced with digitised/georeferenced maps, from which the polygons of man-made structures and natural elements can be extruded, with the aim of exponentially increasing the accuracy of the 3D model of the reconstructed landscape.

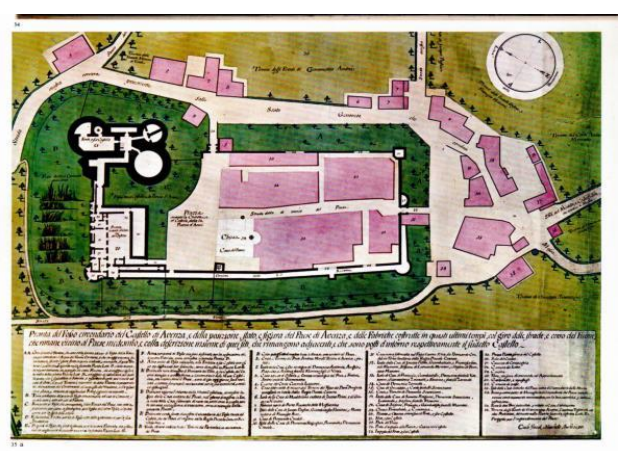

Figure 3. Avenza Castle Map, 1795 (ASMo)

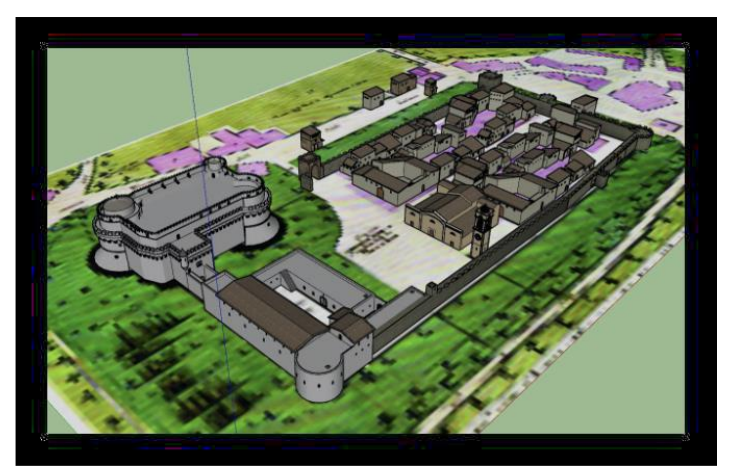

Figure 4. Example of polygons extrusion to generate 3D buildings in a virtual landscaping reconstruction before 